\title{
SUSTAINED AND TRANSIENT NEURAL MODULATIONS IN PREFRONTAL CORTEX RELATED TO DECLARATIVE LONG-TERM MEMORY, WORKING MEMORY, AND ATTENTION
}

\author{
Petter Marklund ${ }^{1}$, Peter Fransson ${ }^{2}$, Roberto Cabeza ${ }^{3}$, Karl M. Petersson ${ }^{4}$, Martin Ingvar ${ }^{2}$ and Lars Nyberg $^{5,6}$ \\ $\left({ }^{1}\right.$ Department of Psychology, Umeå University, Umeå, Sweden; ${ }^{2}$ Cognitive Neurophysiology Research Group, \\ Department of Clinical Neuroscience, Karolinska Institute, Stockholm, Sweden; ${ }^{3}$ Center for Cognitive Neuroscience, \\ Duke University, Durham, NC, USA; ${ }^{4}$ Cognitive Neurology and Memory Research Group, F.C. Donders Centre for \\ Cognitive Neuroimaging, Radboud University of Nijmegen, Nijmegen, The Netherlands; ${ }^{5}$ Department of Radiation \\ Sciences, Diagnostic Radiology, Umeå University, Umeå, Sweden; ${ }^{6}$ Department of Integrative Medical Biology, \\ Physiology Section, Umeå University, Umeå, Sweden)
}

\begin{abstract}
Common activations in prefrontal cortex (PFC) during episodic and semantic long-term memory (LTM) tasks have been hypothesized to reflect functional overlap in terms of working memory (WM) and cognitive control. To evaluate a WM account of LTM-general activations, the present study took into consideration that cognitive task performance depends on the dynamic operation of multiple component processes, some of which are stimulus-synchronous and transient in nature; and some that are engaged throughout a task in a sustained fashion. PFC and WM may be implicated in both of these temporally independent components. To elucidate these possibilities we employed mixed blocked/event-related functional magnetic resonance imaging (fMRI) procedures to assess the extent to which sustained or transient activation patterns overlapped across tasks indexing episodic and semantic LTM, attention (ATT), and WM. Within PFC, ventrolateral and medial areas exhibited sustained activity across all tasks, whereas more anterior regions including right frontopolar cortex were commonly engaged in sustained processing during the three memory tasks. These findings do not support a WM account of sustained frontal responses during LTM tasks, but instead suggest that the pattern that was common to all tasks reflects general attentional set/vigilance, and that the shared WM-LTM pattern mediates control processes related to upholding task set. Transient responses during the three memory tasks were assessed relative to ATT to isolate item-specific mnemonic processes and were found to be largely distinct from sustained effects. Task-specific effects were observed for each memory task. In addition, a common item response for all memory tasks involved left dorsolateral PFC (DLPFC). The latter response might be seen as reflecting WM processes during LTM retrieval. Thus, our findings suggest that a WM account of shared PFC recruitment in LTM tasks holds for common transient item-related responses rather than sustained state-related responses that are better seen as reflecting more general attentional/control processes.
\end{abstract}

Key words: prefrontal cortex, sustained/transient, declarative long-term memory, attention, working memory, cognitive control

Functional neuroimaging of human declarative (episodic and semantic) long-term memory (LTM) has revealed commonalities among recruited brain regions across a wide array of tasks, preferentially involving prefrontal cortex (PFC) (Cabeza and Nyberg, 2000). A hypothesis is that PFC regions similarly engaged in episodic and semantic LTM tasks may be related to working memory (WM) operations and cognitive control processes (Wagner, 1999, 2001). In line with such a proposal, direct comparisons of brain activity associated with LTM and WM tasks have consistently demonstrated overlapping PFC activations (Cabeza and Nyberg, 2000; Braver et al., 2001; Cabeza et al., 2002; Ranganath et al., 2003, 2004, 2005; Nyberg et al., 2003). However, other functional neuroimaging studies have revealed overlap in neural activity between visual attention (ATT) tasks and different memory tasks, i.e. episodic recognition (Cabeza et al., 2003), verbal working memory (Coull et al., 1996; LaBar et al., 1999) and spatial working memory (see Awh and Jonides, 2001 for a review). In particular, a close relationship has been established between WM and ATT with regard to common fronto-parietal engagement, which implicate shared cognitive mechanisms (Desimone and Duncan, 1995; Awh and Jonides, 2001). Hence, it remains unclear to what extent findings of commonly recruited prefrontal areas during different LTM tasks may reflect WM processes or more basic attentional mechanisms related to maintaining an attentive state of "readiness" throughout task performance.

Previous such cross-function investigations of regional activation similarities have typically relied on either blocked or event-related paradigms that provide measures of task-induced activity that differ regarding the timescale of the neural responses they account for. Mixed designs that combine blocked and event-related functional magnetic resonance imaging (fMRI) procedures can dissociate the relative contributions of temporally independent neural modulations (Düzel et al., 1999; Donaldson and Buckner, 2001; Visscher et al., 2003), by separating responses that are sustained throughout the task from responses that are 
transient in nature (i.e., stimulus-synchronous) (c.f., Düzel et al., 1999).

Sustained responses are thought to subserve state-related processing, putatively induced by task instructions to direct behaviourior in a contextrelevant manner during the entire task. Two key component processes that may elicit sustained neural modulations are top-down attentional biasing and cognitive control. First, some sustained responses likely support the maintenance of an enhanced level of alertness and attentional focus throughout task performance (Posner and Petersen, 1990; Coull, 1998). Second, certain processes such as maintaining the relevant task set is likely shared by many cognitive tasks (Wagner, 2001; Braver and Barch, 2002), and such processes may be termed task-general WM processes.

Transient responses reflect mechanisms that support item-related processing per se. Item processes may involve components such as stimulus coding, retrieval control during memory search (Wagner, 2001; Buckner, 2003), response selection (Thompson-Schill et al., 1999) as well as motor execution.

Common prefrontal activations may reflect either sustained or transient neural responses. Here we used "mixed" event-related/blocked fMRI procedures to index sustained and transient bloodoxygen-level-dependent (BOLD) signal changes during four cognitive tasks, episodic yes/no recognition, semantic classification, 2-back WM, and sustained ATT. For sustained brain activity, a primary aim was to investigate whether staterelated responses would generalize across episodic and semantic LTM tasks. Furthermore, in an attempt to examine the presumably shared cognitive mechanisms underlying overlapping sustained responses, we conducted additional analyses of regions exhibiting common sustained activity during the LTM tasks in relation to sustained responses elicited during WM and ATT tasks. Patterns of sustained activity increases common to all tasks might reflect general attentional processes. Sustained activity associated with the LTM and WM tasks, but not the ATT task, might reflect task set and preparatory processes to ensure context-appropriate processing once a stimulus item is presented. Moreover, some sustained responses were expected to differentiate WM and ATT from LTM tasks because the latter required no item processing in-between test items (inter-stimulus intervals - ISIs - varying between 3-24 sec), whereas active on-line processing was continuously required during the ATT and WM tasks.

For transient brain activity, the LTM and WM tasks were contrasted with the ATT task to isolate item-specific mnemonic processes from visual and motor activity. We expected some transient frontal responses to generalize across all memory tasks, including dorsolateral PFC (DLPFC) activity
(Courtney et al., 1997; Cabeza et al., 2002). Other frontal responses were expected to be recruited in a task-specific manner; with differential activity in right ventrolateral PFC (VLPFC) for episodic memory, left VLPFC for semantic memory, and premotor cortex for WM (Nyberg et al., 2002).

\section{Materials AND Methods}

\section{Subjects}

Fourteen healthy adults volunteered to participate in the experiment, one of which had to be excluded from further analysis due to technical problems with the data acquisition. Of the remaining 13 subjects (5 male, 8 female; mean age: 28 years, range: 22-41 years), the behaviouralioral data from one subject was incomplete, with only three out of four functional sessions completed. This subject was included in all statistical analyses, except for region of interest (ROI) time course analyses. Thus, the randomeffects analyses were based on 13 subjects, and the ROI time course analyses were based on 12 subjects. Subjects gave written informed consent. All were right-handed and had normal or correctedto-normal visual acuity. None of the subjects had a history of neurological or psychiatric illness. The study was approved by the local ethics committee at the Karolinska Hospital.

\section{Task Procedures}

Before the subjects were placed in the scanner, they were presented with a study list of 40 words with the instruction to memorize as many as possible during two successive presentations of the same list $(2.5 \mathrm{sec} /$ word $)$. For each subject, 12 of the studied words were randomly chosen as targets for the subsequent recognition tests in the scanner. Subjects were given instructions regarding all task procedures and performed a short pre-scan practice run to ensure that task instructions were properly understood.

Each of the four functional runs included four task conditions, each represented in task blocks of $90 \mathrm{sec}$. The task blocks were separated by resting blocks (25 sec) during which subjects were instructed to rest while fixating a small circle that was constantly displayed at the centre of the screen. Functional runs were initialized after the presentation of a visual prompt $(10 \mathrm{sec})$, followed by another visual prompt $(2.5 \mathrm{sec})$ instructing the subjects to "REST", and subsequently the small circle was shown for $25 \mathrm{sec}$. After this initial rest period, the first task was initiated by the presentation of a visual prompt $(2.5 \mathrm{sec})$ that informed subjects of which task was to be performed (Figure 1). The prompts initializing respective task were: RECOGNITION (episodic 


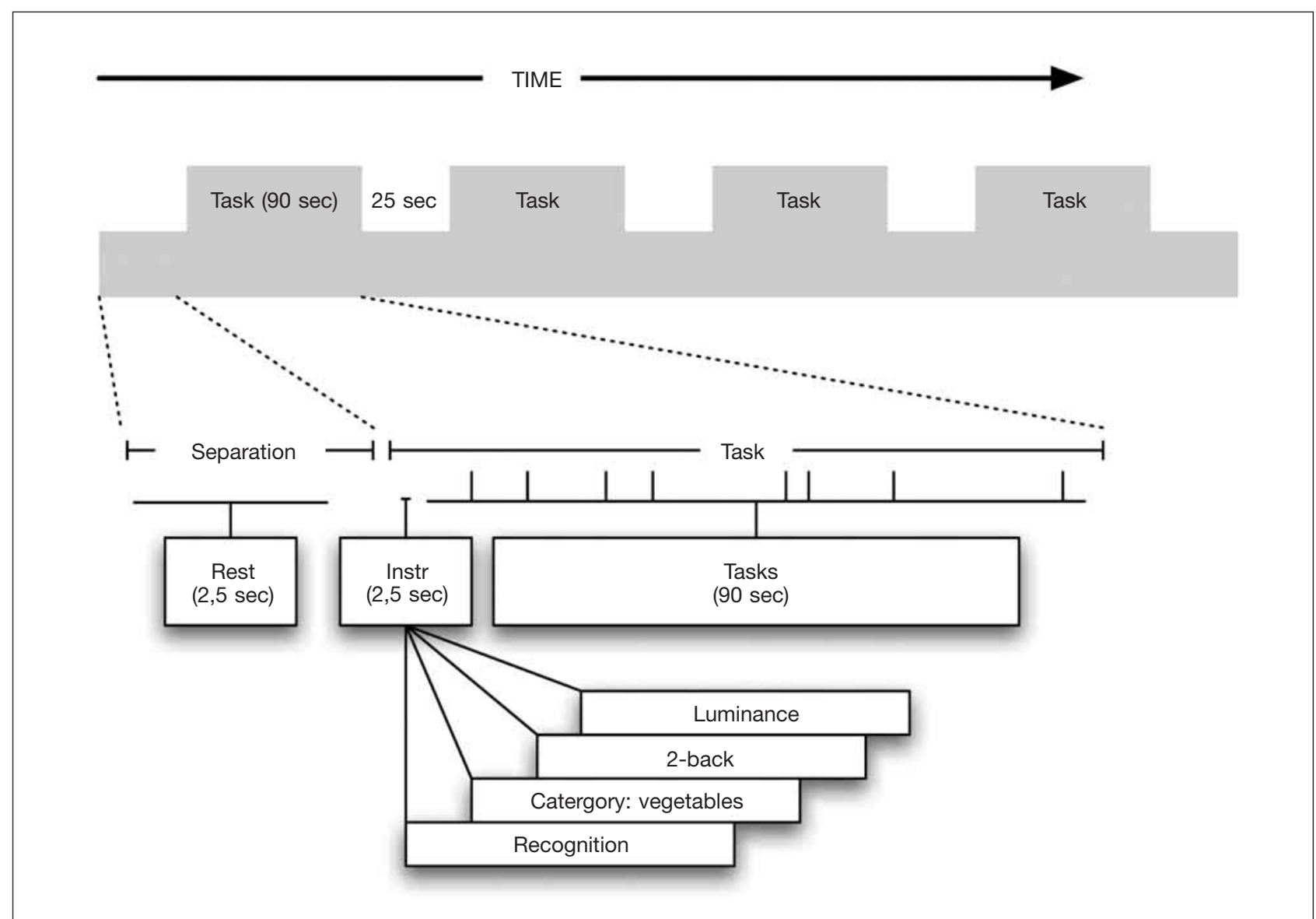

Fig. 1 - Schematic illustration of the mixed blocked/event-related functional magnetic resonance imaging (fMRI) paradigm used in the present study. In each functional run, the four task blocks were separated by resting blocks. A task instruction initiated each task block, within which individual items were intermixed with fixation gaps of varying time duration.

task), CATEGORY, and the specific category target, e.g. VEGETABLES (semantic categorization task), 2-BACK (WM task), and LUMINANCE (ATT task). The order of the four task conditions within each functional run was counterbalanced across runs within subjects and across subjects. Task conditions were also counterbalanced across subjects such that each condition followed every other condition equally often.

The visual stimuli (black letters on white background) were presented using the ERTS software (J. Beringer, BeriSoft Cooperation, Frankfurt, Germany, 1987) and projected onto a screen positioned at the foot end of the scanner bore. Subjects comfortably viewed the screen through a mirror attached to the head coil. An MRI-compatible response box was attached to the right-hand of the subjects and used to record task performance (reaction times - RT - and accuracy).

\section{Experimental Tasks}

The study included four conditions: episodic LTM retrieval, semantic LTM retrieval, WM, and sustained ATT. The tasks used identical stimuli (visual presentation of single words), with the exception of the sustained ATT task (which included a single letter string). Across memory tasks, each task block included 8 individual items, each presented for $2.5 \mathrm{sec}$ in a jittered manner. Word items were intermixed with a fixation crosshair at the centre of the screen of pseudorandom duration. The cross-hair was continuously displayed throughout each task condition, with single words shown just below. Task conditions were identical with respect to behavioural response (key-press 'yes/no'), except for the ATT task (keypress 'yes' only). The item-type ratio in the memory tasks was $3 / 8$ for trials requiring a "yes"response and 5/8 for trials requiring a "no"response. This disproportionate relation between items was due to the particular nature of 2-back paradigms, where the two first items by necessity produce "no"-responses. The ATT task required subjects to respond "yes" at each of eight instances of target detection (see below).

For the episodic retrieval task, we used a "yes/no" recognition paradigm that required subjects to press a specified "yes" button with the index finger of the right hand each time they recognized the presented word as "old" (i.e., part of a pre-scan study list), and to press a specified "no" button with the ring finger of the right hand to indicate that the word was "new" (i.e., not part of the pre-scan study list). To assess semantic retrieval we used a category classification task. The 
task required subjects to decide whether each presented word was a subordinate concept of a specific target category or not (the categoryinstance used as target was visually presented at the start of a semantic task block). Subjects were instructed to press "yes" each time they identified a word as a subordinate of the target category and to press "no" if the word did not fit the category. Different category instances were used for each of the four semantic task blocks. The non-category words used were members of a single category semantically close to the target category (e.g., fruit $v s$. vegetables). For the WM condition a 2-back task paradigm was used (Kirchner, 1958). Subjects were instructed to press "yes" each time they thought the presented word was the same as the word presented two items earlier in the sequence, and to press "no" each time it was not. We used a vigilance paradigm (Mackworth, 1957) to assess sustained ATT, during which subjects were instructed to maintain focal ATT at six black lettered $X^{\prime}$ s in a row (XXXXXX) that were continuously displayed for the duration of the task. At jittered intervals the luminance of the stimulus was slightly dimmed for $400 \mathrm{msec}$ and the subjects were required to press a button with the index finger each time they perceived a stimulus dimming. This stimulus change occurred 8 times in each ATT task block. By using a very subtle change in luminance, the sustained attention/vigilance demand was kept high.

\section{MR Image Acquisition}

Structural T1-weighted images $(\mathrm{TR} / \mathrm{TE} / \mathrm{flip}=24$ $\mathrm{msec} / 6 \mathrm{msec} / 35^{\circ}, .9 \times 1.5 \times .9 \mathrm{~mm}^{3}$ voxel size $)$ and functional echo-planar images (pixel size $=3.4$ $\times 3.4 \mathrm{~mm}^{2}$, TE $=40 \mathrm{msec}$ ) with BOLD contrast were acquired on a General Electric Signa Echo Speed 1.5T MR scanner. Each echo-planar image volume comprised 25 slices (thickness $=5 \mathrm{~mm}$, slice gap $=.5 \mathrm{~mm}$ ) covering the whole brain except for the most inferior part of the cerebellum. The echo-planar image acquisition sessions consisted of 194 volumes acquired continuously with a repetition time (TR) of $2.5 \mathrm{sec}$. Four sessions were performed in each subject and each session was preceded by four "dummy" scans to allow for $\mathrm{T} 1$ equilibrium effects. The duration of each functional run was $8 \mathrm{~min}$ and $5 \mathrm{sec}$. Four runs were acquired per subject with 5-10 minutes in between.

\section{Data Analysis}

Behavioural task performance was analyzed to assess differences in difficulty across the four task conditions by considering accuracy and RT measures.

Functional imaging data were pre-processed in several steps prior to statistical analyses using the
SPM99 software package (http://www.fil.ion.ucl.ac. $\mathrm{uk} / \mathrm{spm} / \mathrm{spm} 99 . \mathrm{html}$ ). Image realignment to the first volume was performed using a truncated sinc interpolation. Subsequent to realignment, all image volumes were corrected for variability in slice timing acquisition, normalized to an approximate Talairach space (Talairach and Tournoux, 1988) as defined by the SPM99 T1-weighted MNI template, and finally smoothed with an isotrophic $10-\mathrm{mm}$, full-width, half-maximum (FWHM) Gaussian kernel.

In mixed blocked/event-related designs the regressors modelling item-related responses will invariably be correlated to some degree with their corresponding state-related regressors. A high degree of correlation implies a low parameter estimation efficiency (Otten et al., 2002; Visscher et al., 2003). In this study, we sought to minimize the degree of correlation between item and state regressors by introducing a jittering of the intervals between consecutive items within each state block. The ISIs were pseudorandomized between 3 and $28 \mathrm{sec}$ according to an approximate Poisson distribution (i.e., for $61 \%$ of the items, the ISI was $3 \mathrm{sec}$, for $30 \%$ of the items, the ISI was $15 \mathrm{sec}$, and only for a minority of items, ISIs were 20-25 sec long) such that the correlation between item and state-related regressors did not exceed .6. This degree of correlation allows a reasonably efficient estimation of item- and state-related activity, while at the same time not compromising the test properties.

Sustained and transient effects were separately modelled in the framework of the general linear model (GLM) (Friston et al., 1995) as implemented in SPM. In detail, item-related transient responses were modelled as regressors containing delta functions representing onset of stimulus whereas state-related sustained responses where modelled with a boxcar function (Friston et al., 1998). For the ATT task, the item regressor represented the onsets of the luminance change, the duration of which matched that of stimulus presentations in the memory tasks. Both regressor-types were convolved with a canonical hemodynamic response function. All data were low-pass filtered (temporal Gaussian kernel, FWHM = $4 \mathrm{sec}$ ) as well as highpass filtered (cut-off frequency $=.002 \mathrm{~Hz}$ ) by including low frequency regressors in the GLM as covariates of no interest.

Applying the GLM to the data resulted in least square estimates of all regressors on a subjectspecific level averaged across the four sessions. Effects of interest were calculated as linear combinations of the individual regressors and stored as subject-specific contrast images. All statistical inferences were based on contrast images (statistical parametrical maps, SPMs) from all thirteen subjects that were entered into a secondlevel model (one sample t-tests) for each effect of interest, yielding a random effects analysis (Holmes and Friston, 1998). 
SPMs were generated for both sustained (state) and transient (item) neural activity changes in all task conditions. Statistically significant BOLD signal changes were identified for each task condition relative to rest (sustained effects) and relative to ATT (transient effects). For the transient effects different item response-types (i.e., hits, misses, correct rejections, and false alarms) were collapsed within each task condition and analyzed together. Unless otherwise specified, the False Discovery Rate (FDR) correction for multiple comparisons (Genovese et al., 2002), thresholded at $\mathrm{p}<.05$, was used, and extent threshold was set to 8 voxels. To identify common responses across sets of task conditions, we performed conjunction analyses (Friston et al., 2005; Nichols et al., 2005) using a threshold of $\mathrm{p}<.001$ uncorrected. This method, referred to as "the Minimum Statistic compared to the Conjunction Null" (Nichols et al., 2005), differs from prior methods of conjunction analysis by asserting that all contrasts involved were individually significant at the predefined threshold. The analytical strategy for common sustained effects was to identify activated regions for the LTM tasks, and then relate these to WM and/or ATT. First, a conjunction was performed on the semantic and episodic LTM tasks. Next, the resulting activation map was used to formally assess the relative degree of overlapping sustained activity across tasks. Centred at each peak coordinate identified from the LTM conjunction, a sphere with an 8 -mm radius was created. Within each of these common LTM regions we examined the magnitude of sustained activity elicited during the WM and ATT task, respectively (reported peak activations were thresholded at $\mathrm{p}<.05$ FDR corrected). In addition, a small volume correction (SVC) procedure was used to formally identify the number of activated voxels within an $8 \mathrm{~mm}$ sphere (comprising 257 voxels) of the peak foci of the LTM conjunction with respect to the WM and ATT tasks (a threshold of $\mathrm{p}<.05$ FDR corrected was used for identification of voxels activated above baseline). For the assessment of transient effects relative to ATT, each activation map was inclusively masked with the main effect of corresponding memory condition relative to baseline, thresholded at $p<.001$ uncorrected, in order to ensure that taskinduced transient increases were not caused by relative decreases during the ATT task. For common memory-related transient effects, we performed a conjunction of the three memory tasks relative to ATT, and inclusively masked the overall activation map with the main effect of each memory task relative to baseline, thresholded at $\mathrm{p}<.001$ uncorrected. To illustrate the temporal profiles of sustained and transient activity changes, time course data was extracted, averaged across individuals and plotted for a number of relevant regions $(8-\mathrm{mm}$ radius-spheres centred at peak foci) that were identified from selected contrasts.

\section{RESULTS}

\section{Behavioural Data}

Accuracy across the four tasks was consistently high, and ranged from $.85, \mathrm{SD}=.11$ (episodic task) to $.98, \mathrm{SD}=.03$ (ATT task). In general, performance was less accurate on the LTM tasks (semantic task: .87, SD = .07) than the WM (.96, $\mathrm{SD}=.05)$ and ATT tasks. A repeated-measures analysis of variance (ANOVA) of the accuracy data revealed that the differences were significant $[\mathrm{F}(3$, $12)=14.5 ; \mathrm{p}<.001]$. Pairwise post-hoc comparisons showed that subjects performed significantly worse on the semantic task relative to ATT $(\mathrm{p}<.001)$ and WM $(\mathrm{p}<.001)$, respectively. The episodic task was also associated with significantly less accurate performance relative to ATT $(\mathrm{p}<.007)$ and WM $(\mathrm{p}<.015)$. There was no difference in accuracy between the two LTM tasks. Performance accuracy was also equivalent between the ATT task and the WM task. An ANOVA on mean RTs revealed significant differences between the tasks $[\mathrm{F}(3,12)=52.6 ; \mathrm{p}<.001]$. The fastest RTs were produced during the WM task, which was associated with a significantly faster response than all other tasks (mean $=1215 \mathrm{msec}, \mathrm{SD}=514$, all pairwise post-hoc comparisons relative WM yielded $\mathrm{p}$ values $<.001)$, whereas the ATT task was associated with a reliably slower response as compared to all other tasks (mean $=1592 \mathrm{msec}$, $\mathrm{SD}=159 \mathrm{msec}$, all pairwise post-hoc comparisons relative ATT showed $p$ values $<.001$ ). RTs did not differ between the episodic and semantic memory tasks (episodic task: mean $=1370 \mathrm{msec}, \mathrm{SD}=468$ msec; semantic task: mean $=1418 \mathrm{msec}, \mathrm{SD}=485$ msec, $\mathrm{p}=.7$ ).

\section{Neuroimaging Data}

Blocked and event-related fMRI signal change was used to examine sustained and transient neural responses associated with episodic memory, semantic memory, WM, and sustained ATT. Positive, as well as negative BOLD signal changes (relative to resting baseline for sustained responses and relative to ATT for transient responses) are reported in separate sections.

\section{Sustained BOLD Increases}

Brain regions associated with sustained processing in each task are shown in Figures $2 \mathrm{a}-2 \mathrm{~d}$ and peak foci are listed in Table I. As can be seen from Figure 2, the activity pattern was quite similar for the LTM tasks, albeit with more extensive activation for semantic memory. Direct contrasts showed that there were no significant differences between episodic and semantic memory tasks $(\mathrm{p}>.05)$. A conjunction analysis, where the resulting activation map was inclusively masked 


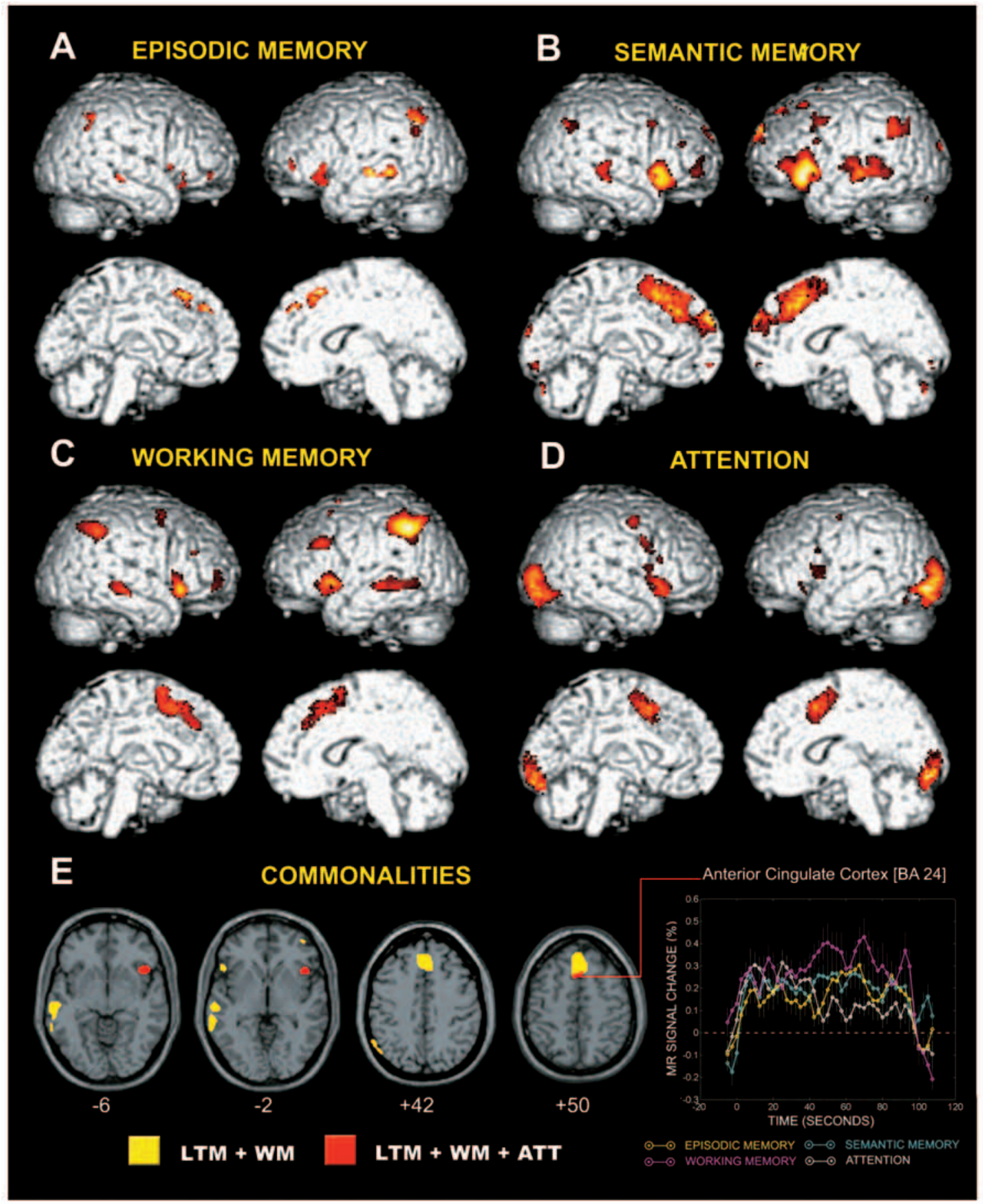

Fig. 2 - Sustained response increases during (a) episodic memory, (b) semantic memory, (c) working memory, and (d) attention, relative to baseline. Statistical parametric maps are superimposed on lateral and medial rendered views of the brain (for coordinates see Table I). Figure $2 a$ was thresholded at $p<.001$ uncorrected; Figures $2 b-2 d$ were thresholded at $p<.05$ false discovery rate (FDR) corrected, extent threshold a-d $>8$ voxels. (e) To visualize the overlaps in sustained activity between long-term memory (LTM), working memory (WM) and attention (ATT), we performed conjunction analyses of the LTM tasks and the main effects of WM and ATT, respectively (thresholded at $p<$ 001 uncorrected). Overlapping sustained response increases for episodic and semantic LTM, WM and ATT are mapped on horizontal sections of a canonical brain (z-coordinates are given for each section). Yellow areas denote regions that showed significant sustained increases across both LTM and WM tasks, but not ATT. Red areas indicate regions that were associated with significant sustained increases across all four tasks including ATT (for coordinates, see Table II). Average time course is depicted for a common dorsal anterior cingulate cortex (ACC)/pre-supplementary motor area (pre-SMA) region (MNI-coordinates; $x=2, y=24, z=50$ ).

with both LTM main effects, showed that overlapping activity increases were located in several distinct PFC subregions including bilateral mid-VLPFC (BA 47/45), frontopolar cortex (BA
10) and medial PFC including the presupplementary motor area (pre-SMA) (BA 6) and a caudal portion of the dorsal anterior cingulate cortex (ACC) (BA 32). In addition, regions in 


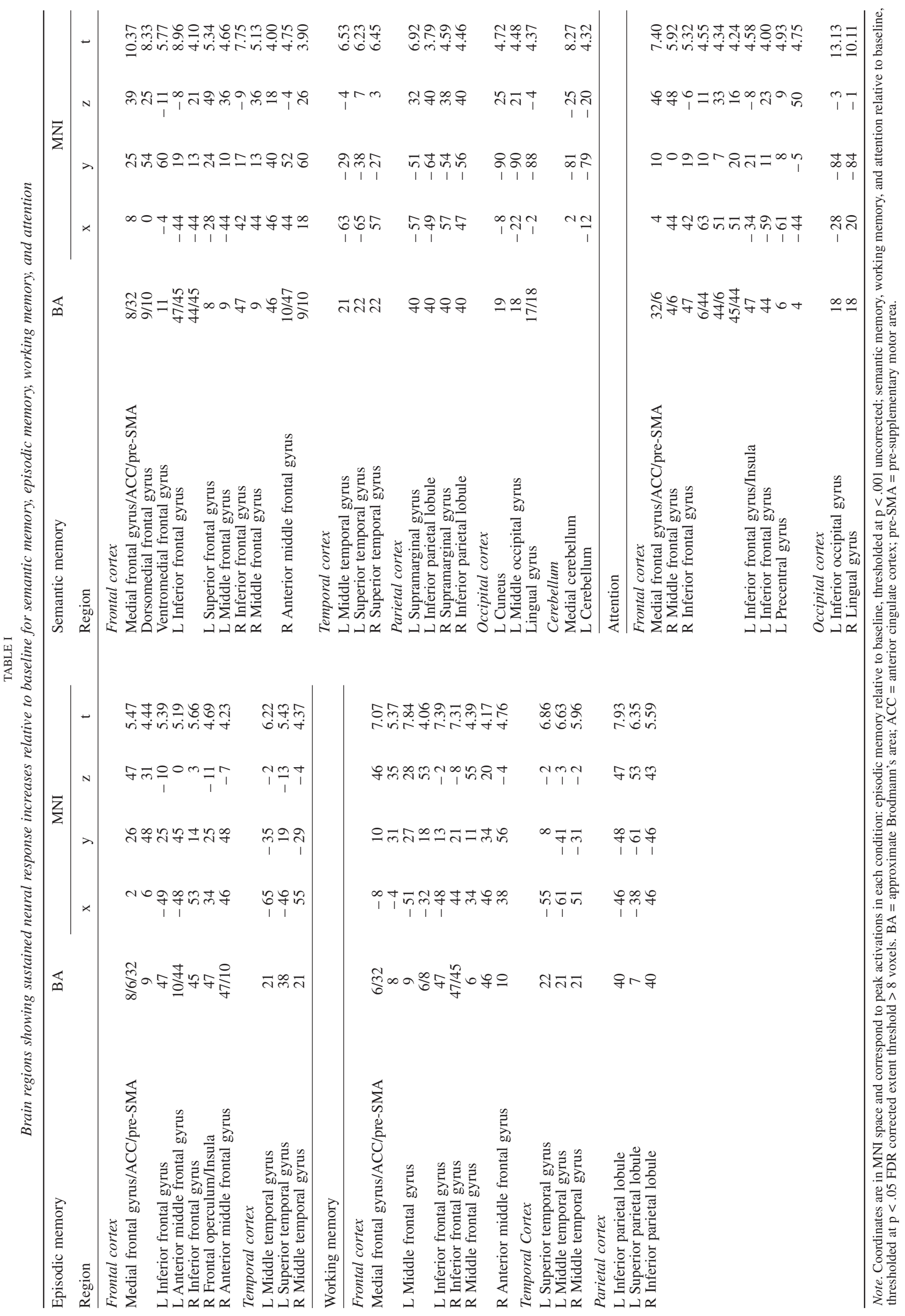




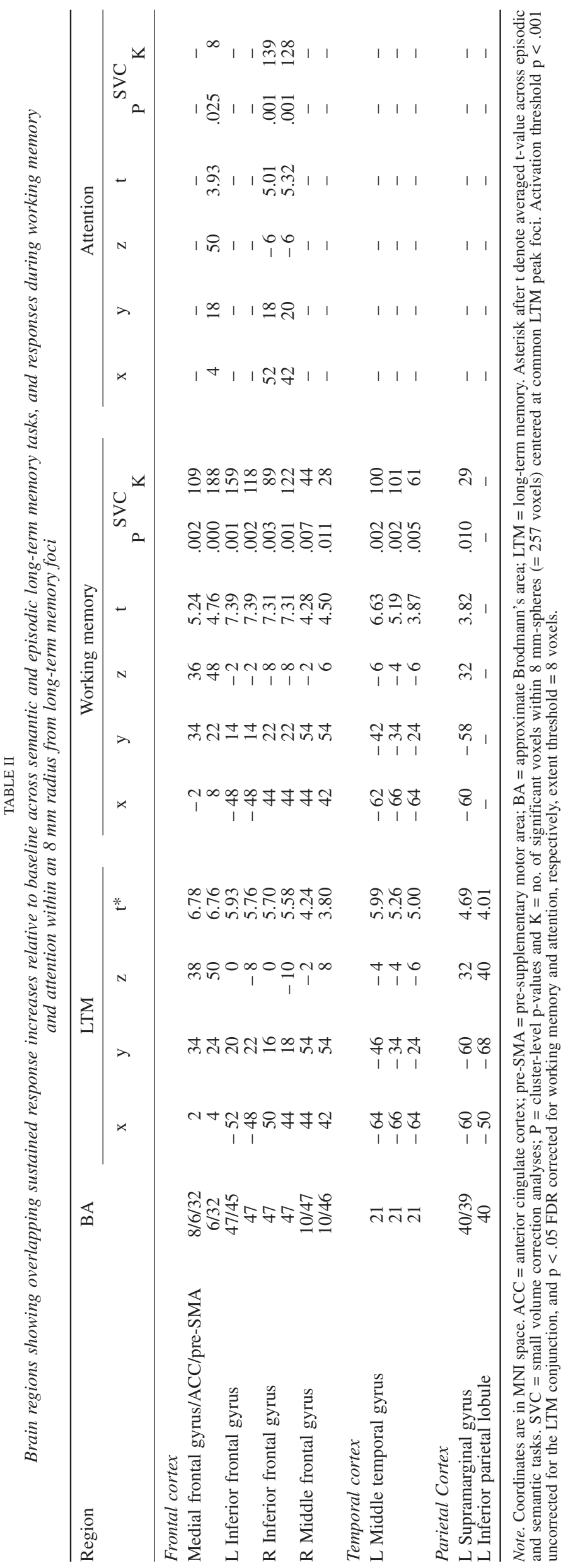


temporal and parietal cortex exhibited LTM-general sustained increases (Table II). The findings of state-related frontal engagement corroborate previous findings of sustained activity patterns in studies using mixed blocked/event-related fMRI designs (Donaldson et al., 2001; Burgund et al., 2003; Velanova et al., 2003), which have shown state-related activity increases in very similar structures.

The coordinates from the conjunction analysis of the LTM tasks were used to create $8-\mathrm{mm}$ spheres surrounding the peak foci to examine whether these regions also were activated during the WM and ATT tasks. This analysis showed that most of the regions associated with LTM were also engaged during the WM task (Table II). A subset of the frontal regions that were commonly activated for the LTM and WM tasks were also activated during the ATT task (Table II) (Figure 2e). These were located in right VLPFC and ACC/pre-SMA and shared a similar sustained activation profile (Figure 2e). Conversely, none of the memory-general regions located in posterior cortices were significantly activated in the ATT task.

Conjunction analyses were done to verify the above results of task commonalities. For the analysis of common regions across all four tasks as well as the analysis of regions that were engaged during the memory tasks but not the ATT task, consistent results were obtained. The only exception was that the conjunction analysis suggested that the left VLPFC region was commonly engaged during all four tasks and not specific to the memory tasks.

\section{Sustained BOLD Signal Decreases}

Brain regions showing relative decreases in sustained BOLD responses (or state-related deactivations) compared with rest, are presented in Figures 3a-3d. As can be seen, the pattern varied substantially across conditions with only a few significant decreases for the LTM tasks, whereas extended decreases were noted for the $\mathrm{WM}$ and ATT tasks, involving practically the whole brain. For both the WM and ATT task, the strongest decreases were located in posterior brain areas.

To investigate commonalities in sustained activity decreases across all task conditions we conducted a conjunction analysis for all main effects relative to baseline, where we inclusively masked the resulting overall deactivation pattern with each main effect. Overlapping decreases were found in medial posterior cortices in the vicinity of the parietooccipital sulcus, including the cuneus, precuneus and the occipital gyrus, as well as the posterior cingulate gyrus (Figure $3 \mathrm{e}$ ). These findings converge with numerous previous studies that have reported similar task-induced decreases relative to low-level baselines (Shulman et al., 1997; McKiernan et al., 2003).

\section{Transient BOLD Signal Changes}

Figures $4 \mathrm{a}-4 \mathrm{c}$ and Table III shows transient activity increases for the LTM and WM tasks relative to ATT. Distinct increases were seen in regions that previously have been associated with these forms of memory. The episodic memory task was associated with increased activity in right VLPFC (BA 47) and left middle temporal cortex (BA 21) extending into medial portions of the temporal lobe (Figure 4a). The semantic memory task was associated with increases in left VLPFC (BA 45) and left lateral occipito-temporal cortex (Figure 4b). The transient activation pattern for the WM task included distinct increases in left DLPFC (BA 9), right premotor (BA 6), and medial parietal cortex (BA 7) (Figure 4c). A conjunction of the LTM and WM tasks relative to ATT, inclusively masked with the main effect of each memory task versus baseline, revealed that common transient increases were located in several discrete regions (see Figure 4d), including left posterior DLPFC in the middle frontal gyrus near the inferior frontal junction rostral to the precentral gyrus (BA 44//9), a small area in left VLPFC (BA 47), and left superior parietal cortex (BA 7) (for coordinates see the figure captions to Figure 4d).

Transient decreases relative to the ATT condition were seen for all tasks, showing the most pronounced effects for the WM task, including several posterior visual regions. As transient decreases were not of primary concern for the present purposes, these results will not be further discussed.

\section{DisCUSSION}

A mixed blocked/event-related fMRI design was used to dissociate sustained and transient responses associated with four different cognitive tasks. For the state-related component, a primary goal was to compare common patterns of sustained neural activation during episodic and semantic LTM tasks with that of WM and ATT. Across all four tasks right VLPFC (BA 47) and medial PFC (BA 6/32) demonstrated sustained recruitment. The engagement of these regions during the attention/vigilance task and during ISIs in the three memory tasks could relate to the apprehension of upcoming events and the operation of a common ATT network (Pashler et al., 2001). Vigilance typically activate right frontal areas (Cohen et al., 1988; Pardo et al., 1991). Right VLPFC and ACC/pre-SMA have been associated with task preparation and ATT in cueing paradigms (Gitelman et al., 1999; Luks et al., 2002; Brass and von Cramon, 2002; Curtis et al., 2004), and ACC is typically considered as part of an anterior attentional network (Posner and Petersen, 1990). Here it should be noted, though, that the 


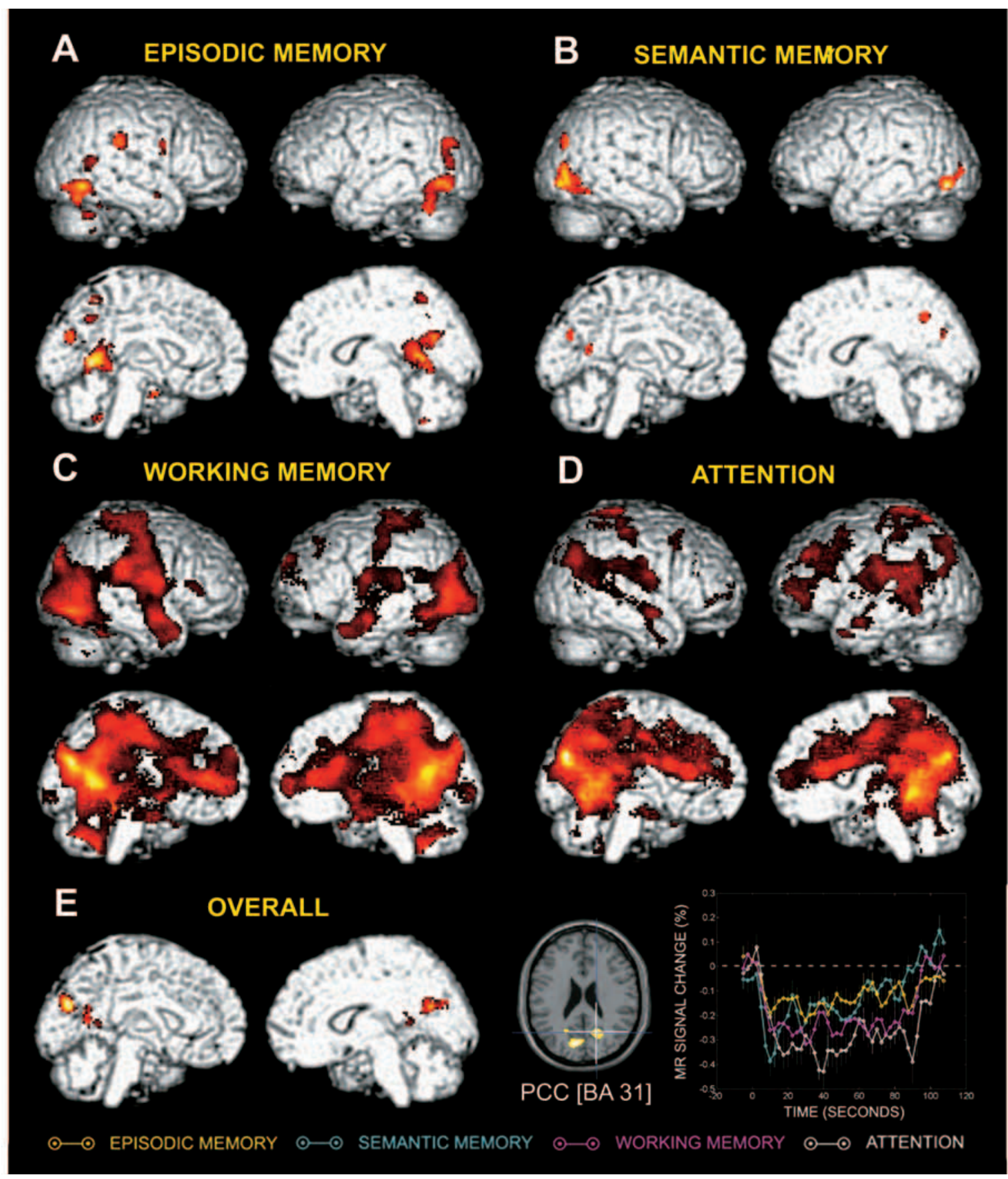

Fig. 3 - (a) Regions associated with sustained response decreases during episodic memory relative to baseline included bilateral posterior cingulate gyrus (BA 29/30/23, MNI-coordinates: $x, y, z ;-10,-48,10$ and $8,-44,17)$, right lingual gyrus (BA 19; 22, -60, $-2)$, right fusiform gyrus $(B A 18 ; 38,-68,-20)$, bilateral middle occipital gyrus (BA 19; -46, - 66, -8 and 50, -62, - 10), left parahippocampal gyrus $(B A 34 ;-30,-2,-26)$, left middle temporal gyrus (BA 39; - 42, - 76, 28), right superior temporal gyrus (BA 22; 56, - 56, 12), right middle temporal gyrus (BA 21; 60, 0, -18), right inferior parietal cortex (BA 40; 68, - 32, 30), precuneus (BA $7 ;-6,-58,38)$, right inferior frontal gyrus/precentral gyrus $(B A 44 / 6 ; 48,4,26)$ and bilateral cerebellum $(-38,-58,-28$ and 30 , -54, -46). (b) Sustained response decreases during semantic memory relative to baseline were observed in bilateral middle occipital gyrus (BA 19/37; - 36, - 74, - 10 and 38, - 70, - 10), precuneus $(B A 7 ; 16,-58,42$ and BA 18; - 2, - 74, 26) and left posterior cingulate gyrus (BA 23; - 18, - 58, 14). (c) Sustained response decreases during WM relative to baseline were observed in the parietooccipital sulcus/precuneus (BA 18; - 8, - 74, 24), left posterior cingulate gyrus (BA 29/30; - 12, - 50, 8), left parahippocampal gyrus (BA 35/36; - 28, - 36, - 14), left inferior frontal gyrus (BA 11/47; - 40, 34, - 16), bilateral cerebellum $(-12,-54,-46$ and $12,-54$, $-48)$, ACC (BA 24/32; - 4, 38, 12), left precentral gyrus (BA 6; - 60,-2, 12), left postcentral gyrus $(B A 3 / 1 ;-46,-20,60)$ and right posterior cingulate gyrus $(B A 23 ; 10,-24,23)$. (d) Sustained response decreases associated with attention relative to baseline were found in left parieto-occipital sulcus (BA 18/19; - 6, - 78, 28), left middle temporal gyrus (BA 39; - 30, - 60, 10), left superior temporal gyrus (BA 21/38; - 40, 10, - 30), bilateral cerebellum (-18, - 50, - 42 and 26, - 50, - 42), right parahippocampal gyrus (BA 28; - 16, -14, -14), bilateral precentral gyrus (BA 4; - 16, - 34, 74 and 20, -30, 74), right postcentral gyrus (BA 3/1; 26, - 40, 74), bilateral parietal cortex (BA 7; - 16, -58, 68 and 14, - 52, 72), bilateral hippocampus $(-36,-18,-16$ and 44, -6, -22) and left middle temporal gyrus $(-56,0,-18)$. Figures $3 a, 3 c, 3 d$ were thresholded at $p<.05$ false discovery rate (FDR) corrected, Figure $3 b$ was thresholded at $p<.001$ uncorrected, extent threshold $>8$ voxels. (e) Overlapping sustained decreases common to all tasks relative to baseline were observed in the parieto-occipital sulcus/precuneus/cuneus (BA 18/19), bilateral precuneus/posterior cingulate gyrus (BA 31 and BA 30/29). Common sustained decreases were revealed by a conjunction analysis for all main effects relative to baseline ( $p<.05$ $F W E$ corrected), where the overall deactivation pattern was inclusively masked with each main effect (thresholded at $p<.001$ uncorrected), extent threshold $>8$ voxels. Time courses are shown for an area in posterior cingulate cortex (PCC, BA 31; MNIcoordinates: $x, y, z ; 18,-62,24)$. 


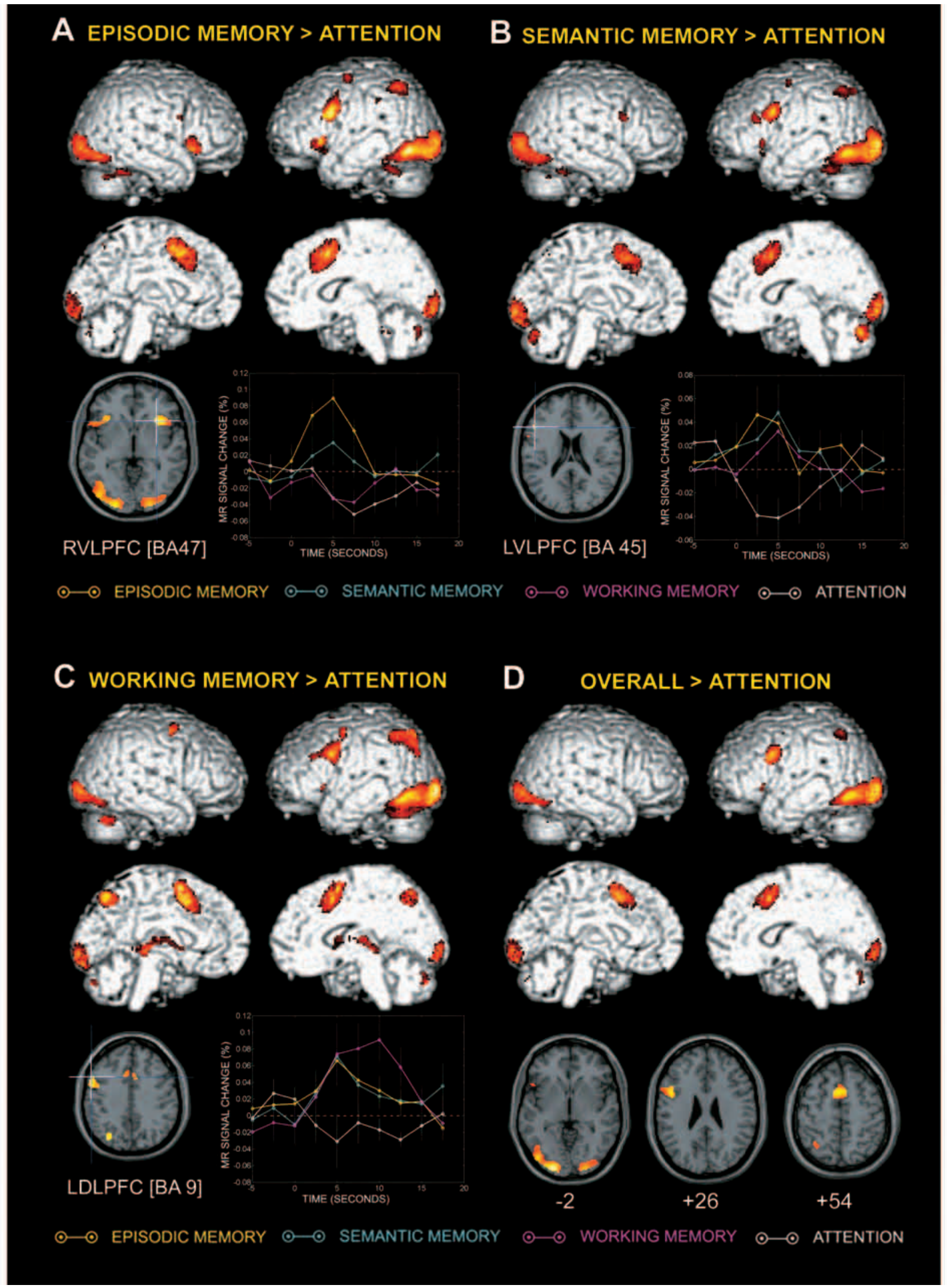

Fig. 4 - (a) Transient response increases for episodic memory relative to attention. Time courses depicted for the right inferior frontal gyrus (BA 47; MNI-coordinates: $x, y, z ; 32,20,-2$ ). (b) Transient response increases for semantic memory as compared to attention. Time courses plotted for a left inferior frontal region (BA 45;-48, 22, 20). (c) Transient response increases for working memory as compared to attention. Time courses plotted for a left middle frontal region (BA 9; - 54, 14, 38). All activations were thresholded at $p<.05$ false discovery rate $(F D R)$ corrected relative to the attention condition, and inclusively masked with the main effect of the corresponding memory condition relative to baseline, thresholded at $p<.001$ uncorrected, extent threshold $>8$ voxels. For coordinates see Table III. (d) Overlapping transient response increases across all memory tasks relative to attention were found in bilateral middle occipital gyrus $(B A 19 ;-42,-68,-10$ and $40,-72,-12)$ and cuneus (BA 18; - 24, - 96, 0 and 24, - 94, 0), anterior cingulate cortex (ACC)/pre-supplementary motor area (pre-SMA) (BA 32/6; - 4, 8, 52), left inferior/middle frontal gyrus (BA 44; - 46, 6, 30, and BA 44/9; - 44, 16, 26), left superior parietal cortex (BA 7; $-32,-58,50)$, medial cerebellum $(6,-80,-34)$ and left inferior frontal gyrus $(B A 47 ;-50,18,2)$. Activations are overlaid on horizontal sections of a canonical brain (z-coordinates are given for each section). 
TABLE III

Brain regions showing transient neural response increase relative to attention for episodic memory, semantic memory, and working memory

\begin{tabular}{|c|c|c|c|c|c|}
\hline \multirow{2}{*}{$\begin{array}{l}\text { Episodic memory } \\
\text { Region }\end{array}$} & \multirow[t]{2}{*}{$\mathrm{BA}$} & \multicolumn{4}{|c|}{ MNI } \\
\hline & & $\mathrm{x}$ & $\mathrm{y}$ & $\mathrm{Z}$ & $\mathrm{t}$ \\
\hline \multirow{2}{*}{$\begin{array}{l}\text { Medial frontal gyrus/ACC/ } \\
\text { pre-SMA }\end{array}$} & $6 / 32$ & 10 & 10 & 48 & 7.99 \\
\hline & 6 & -6 & 6 & 54 & 7.78 \\
\hline L Frontal operculum/Insula & 47 & -32 & 26 & -2 & 5.93 \\
\hline L Inferior frontal gyrus & 47 & -46 & 18 & 2 & 5.37 \\
\hline \multirow[t]{2}{*}{ L Middle frontal gyrus } & 44 & -46 & 8 & 28 & 7.89 \\
\hline & 45 & -42 & 18 & 22 & 7.11 \\
\hline L Middle frontal/Precentral gyrus & $6 / 4$ & -28 & -8 & 62 & 3.81 \\
\hline R Frontal operculum/Insula & 47 & 32 & 20 & -2 & 8.75 \\
\hline R Middle frontal gyrus & 44 & 48 & 8 & 26 & 3.44 \\
\hline \multicolumn{6}{|l|}{ Temporal cortex } \\
\hline R Fusiform gyrus & 37 & 34 & -50 & -22 & 5.15 \\
\hline \multicolumn{6}{|l|}{ Parietal cortex } \\
\hline L Superior parietal lobule & 7 & -32 & -56 & 52 & 6.97 \\
\hline L Inferior parietal lobule & 40 & -40 & -38 & 38 & 3.44 \\
\hline \multicolumn{6}{|l|}{ Occipital cortex } \\
\hline L Middle occipital gyrus & 19 & -40 & -68 & -8 & 10.07 \\
\hline R Middle occipital gyrus & 19 & 38 & -72 & -12 & 7.06 \\
\hline \multicolumn{6}{|l|}{ Cerebellum } \\
\hline Medial cerebellum & & 8 & -78 & -34 & 4.87 \\
\hline R Cerebellum & & 34 & -64 & -32 & 4.03 \\
\hline
\end{tabular}

Semantic memory

Frontal cortex

Medial frontal gyrus/ACC/ pre-SMA

L Middle frontal gyrus

L Inferior frontal gyrus

L Middle frontal/Precentral gyrus $6 / 4-50$

$\begin{array}{llrrrr}\mathrm{R} \text { Inferior frontal gyrus } & 45 & 46 & 14 & 24 & 5.28\end{array}$

Temporal cortex

R Fusiform gyrus

Parietal cortex

L Superior parietal cortex

Occipital cortex

$\begin{array}{lrrrrr}\text { L Cuneus } & 18 & -24 & -98 & 2 & 9.96 \\ \text { R Cuneus } & 18 & 28 & -94 & 4 & 7.07 \\ \text { Cerebellum } & & & & & \end{array}$

Cerebellum

$36-62-32 \quad 3.17$

Working memory

Frontal cortex

Medial frontal gyrus/ACC/ pre-SMA

L Middle frontal gyrus

$\begin{array}{lllll}6 / 8 & 4 & 10 & 56 & 7.28 \\ 32 & 8 & 12 & 42 & 6.56\end{array}$

$\begin{array}{crrrr}32 & 8 & 12 & 42 & 6.56 \\ 44 / 9 & -44 & 6 & 30 & 6.19\end{array}$

al/Precentral gyrus $6 \quad-40 \quad-2 \quad 50 \quad 4.62$

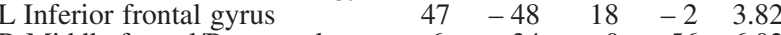

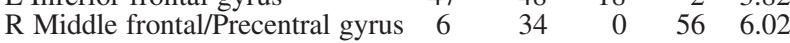

Parietal cortex

$\begin{array}{llllll}\text { L Precuneus } & 19 & -32 & -74 & 40 & 6.39\end{array}$

$\begin{array}{llllll}\text { L Inferior parietal lobule } & 40 & -36 & -54 & 48 & 6.03\end{array}$

$\begin{array}{llllll}\text { Medial precuneus } & 7 & -6 & -62 & 48 & 4.77\end{array}$

Occipital cortex

L Cuneus

R Cuneus

Cerebellum

Medial cerebellum

R Cerebellum

Other structures

Thalamus

Caudate nucleus

$\begin{array}{lllll}18 & -26 & -96 & -2 & 7.49\end{array}$

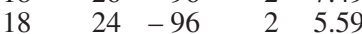

$\begin{array}{llll}4 & -82 & -34 & 5.70\end{array}$

$38-64-32 \quad 5.61$

$\begin{array}{llll}8 & -20 & 6 & 4.24\end{array}$

Note. Coordinates are in MNI space and correspond to peak activations in each condition relative to attention, thresholded at $\mathrm{p}<.05$ FDR corrected, and inclusively masked with corresponding main effect relative to baseline, thresholded at $\mathrm{p}<.001$ uncorrected; extent threshold $>8$ voxels. BA = approximate Brodmann's area; ACC = anterior cingulate cortex; pre-SMA $=$ pre-supplementary motor area. conjunction analysis suggested that bilateral VLPFC regions were engaged across all tasks.

Frontal sustained activity that were common for LTM and WM tasks, in the absence of significant effects during ATT, were located in right frontopolar cortex (BA 10), and a midline area (BA $8 / 6 / 32$ ), rostral to the task-general medial site. An effect was also seen in left VLPFC (BA 47/45), although the conjunction analysis indicated that this region was also engaged during the ATT task. This pattern of sustained activity may represent information about the task context, e.g., instructions, (Petersson et al., 2003) that is necessary to maintain on-line in an active state during the temporal gaps between items in the memory tasks (Cohen et al., 1996). Such ISIs were missing in the ATT task, where a single item was constantly displayed. Activity in right frontopolar cortex and ACC has consistently been noted during episodic retrieval (Cabeza and Nyberg, 2000; Lepage et al., 2000), and such activity has been interpreted as reflecting instantiation and maintenance of a neurocognitive set underlying episodic retrieval (Tulving et al., 1994; Nyberg et al., 1995; Lepage et al., 2000). The present finding of sustained frontopolar and ACC activity during episodic memory retrieval is consistent with this view (c.f., Cabeza et al., 2002), but the fact that such activity was also seen for the semantic and WM tasks indicates a more general functional role. Indeed, frontopolar activation has been associated with WM tasks (Braver and Bongiolatti, 2002; Cabeza et al., 2002; Nyberg et al., 2002), semantic monitoring tasks (MacLeod et al., 1998), and tasks of cognitive branching (Koechlin et al., 1999). A recent study by Sakai and Passingham (2003) also associated activity in the anteriormost region of the frontal lobes with preparatory processes during the period between task instruction and the first memory items to be retained in WM. Furthermore, in a previous fMRI study using mixed blocked/event-related design to explore the functional anatomy and temporal dynamics underlying controlled episodic retrieval (Velanova et al., 2003), a similar set of regions showed sustained activity as in the present study (frontopolar, medial frontal, and parietal cortices).

A striking observation was the extensive decreases in sustained activity, relative to rest, during the WM and ATT tasks (c.f., Shulman et al., 1997; McKiernan et al., 2003). That this effect was much stronger for WM and ATT than the LTM tasks, is likely related to the prolonged high levels of focused ATT and intensive processing demands in the former tasks (c.f., Ghatan, 1995). That is, in contrast to LTM tasks where processing demands diminished during ISIs, WM and vigilance tasks were characterized by continuous processing throughout the entire duration of the task. The most profound cerebral consequence of this persistency in effortful cognitive processing is seemingly 
widespread regional decreases in neural activity. These apparent task-selective deactivations might be related to the differential operation of a default mode of brain activity (Fransson, 2005; Gusnard and Raichle, 2001; Raichle et al., 2001), during ISIs of the LTM tasks. Under resting conditions, neural activity has been revealed in an extensive brain network, including precuneus/posterior cingulate cortex, lateral parietal cortex, and the parieto-occipital sulcus (Raichle et al., 2001). This pattern might reflect a default mode of brain activity that involve processes such as spontaneous thought (Christoff et al., 2004), self-reflection and monitoring of internal states and the external environment (Shulman et al., 1997; Gusnard et al., 2001). Our findings could reflect the reinstantiation of the default mode in the comparatively process vacant ISIs during the LTM tasks. Hence, when item-independent brain responses in the LTM tasks were contrasted with rest, where the default mode should be the main process, this activity was cancelled out. By contrast, in WM and ATT, when there was continuous item-processing throughout the tasks, the default mode was likely disabled. Therefore, when compared with rest, brain regions related to the default mode appeared as deactivated. It should also be noted that selective deactivations were observed in visual areas for the 2-back WM task. Since decreases in visual areas are not part of the "default mode network", these deactivations may reflect a more active suppression of brain activity and processing in regions not engaged in WM maintenance rather than a more passive disengagement related to the default mode. A complementary interpretation as to the relative absence of sustained decreases in the semantic memory-rest comparison is that the default mode during rest predominantly involves conceptual processing. Accordingly, semantic retrieval and resting conditions have been shown to activate similar regions when contrasted with non-semantic tasks (Binder et al., 1999). In a similar vein, default-mode activity has been related to episodic memory processing (Greicius et al., 2004). Taken together, increased and decreased sustained brain activity can be interpreted in terms of control signals that promote top-down biasing of taskrelevant versus irrelevant processing pathways (Desimone and Duncan, 1995; Ghatan et al., 1998; Miller and Cohen, 2001; Braver and Barch, 2002).

In addition to the sustained effects, transient stimulus-synchronous increases were seen for all tasks. These activations generally occurred in areas distinct from the sites of sustained responses, which provide additional evidence that state- and item-related brain activity can be dissociated. One transient activation that was common to all memory tasks was located in left posterior DLPFC (BA 44/9). It is striking that this response was transient as DLPFC often is implicated in relation to representing and maintaining task context
(Cohen et al., 1996; MacDonald et al., 2000; Curtis and D'Esposito, 2003). In contrast, our results implicate that this region is concerned with itemspecific processing. Left posterior DLPFC has shown event-related modulations in previous studies; in the context of episodic retrieval and enhanced control demand (Velanova et al., 2003), retrieval across WM and episodic memory tasks (Cabeza et al., 2002), and transient control during task-switching (Braver et al., 2003). Such activations may reflect general retrieval-control processes (Wheeler and Buckner, 2003) and retrieval from LTM may generate an active representation, similar to that produced during updating of WM-contents (Courtney et al., 1997).

Moreover, differential transient responses were noted for all three memory tasks. Such effects could reflect actual recovery of domain-specific information from episodic, semantic and WM, respectively. For the episodic task, a salient transient effect was observed for right PFC (BA 47). This region has previously been suggested to be part of a network subserving episodic retrieval mode (Nyberg et al., 1995; Lepage et al., 2000). The present observation that this region was associated with item-related processing appears inconsistent with this suggestion and more coherent with interpretations of right prefrontal activity in terms of post-retrieval monitoring (Rugg et al., 1996) and/or cue specification (Henson et al., 1999). However, it is possible that subjects initiated a retrieval mode only when items (i.e., retrieval cues) were presented rather than trying to maintain it across the task block. If true, the fairly long intervals between some cues could have contributed to this behaviour (i.e., participants returned to the default mode in-between test items). For the WM task, transient increases were observed in DLPFC (BA 9), premotor areas (BA 6), and precuneus (BA 7). This pattern is consistent with previous findings from WM studies (Cohen et al., 1997; Nyberg et al., 2002; for a review, see Smith and Jonides, 1997). The specific WM task that we used, 2-back, involves several different itemprocesses that can be related to the observed transient effects, including temporal coding of items (Cohen et al., 1997) and updating functions (Collette and Van der Linden, 2002). The semantic task was associated with transient activations in left VLPFC and left lateral occipito-temporal cortex, which is in keeping with numerous previous studies of semantic memory (Martin, 2001; Thompson-Schill, 2003). One candidate function of left VLPFC is to guide controlled semantic retrieval (Wagner et al., 2001). As our semantic task required the participants to classify items that belonged to conceptually related categories (e.g., fruit-vegetables), this account is consistent with the present observations. It should be noted that the pattern observed for semantic memory largely overlapped those for the episodic and WM tasks, 
which may reflect a task-general verbal processing/semantic component (Braver et al., 2001). A point of consideration concerns the fact that the analyses of transient effects were based on items associated with both correct and incorrect responses, which might have affected the results. However, since the differences in accuracy between conditions were small and relatively few items were used, it is unlikely that this procedure should have had any significant effect on the activation data.

In summary, the present findings present a complex and equivocal picture with respect to a WM account of common PFC activations in episodic and semantic LTM tasks. The subset of overlapping regions that were attributed to more general attentional processes included bilateral, but predominantly right, VLPFC and a posterior part of ACC/pre-SMA. These regions may mediate topdown attentional biasing and vigilance related to the apprehension of upcoming events to be acted upon. Regarding the sustained state-related effects, the patterns of commonalities in frontal engagement that differentiated the three memory tasks from the ATT task involved ACC and frontopolar areas. These regions, which were common to LTM and WM, constitute candidate regions for which a WM account of frontal LTMactivity might apply. However, these specific activation sites are not generally attributed to WM maintenance processes. Instead, in previous work, these regions have been considered a signature of retrieval mode (Lepage et al., 2000), indicating a more generic functional role of the neural circuitry underlying retrieval mode in episodic memory tasks. Frontopolar cortex and ACC/pre-SMA activity have also been linked to anticipatory task preparation and the representation of intention (Sakai and Passingham, 2003; Lau et al., 2004). Collectively, these functional accounts indicate that the sustained state-related effects that were common to WM and LTM may reflect cognitive control processes related to task set in a way that is not dependent on WM maintenance.

Given that overlapping sustained activity changes may reflect general control signals that govern task-relevant processing, a possibility is that a WM account of frontal LTM activity holds for transient activity changes. The region in posterior DLPFC (BA 44/9) that was found to be equally activated across all memory tasks may represent WM processes that coordinate the active retrieval of information from episodic and semantic LTM, which tentatively generalize across a wide range of cognitive tasks. As was discussed above, left posterior DLPFC has shown item-related modulations in previous studies, and Courtney et al. (1997) noted that "activation of the same areas by both long-term retrieval and working memory is consistent with the idea that retrieval produces an active representation of the recalled material much like the active representation of material held during working memory delay" (p. 610).

In conclusion, studies employing decomposition of overlapping and specific neural responses across multiple tasks into sustained versus transient modulations promises to provide a better understanding of the multifaceted relation between PFC subregions and component processes underlying human memory.

Acknowledgements. This research was supported by a grant from the Swedish Research Council.

\section{REFERENCES}

AwH E and Jonides J. Overlapping mechanisms of attention and spatial working memory. Trends in Cognitive Sciences, 5: 119-126, 2001

Binder JR, Frost JA, Hammeke TA, Bellgowan PS, Rao SM and Cox RW. Conceptual processing during the conscious resting state: A functional MRI study. Journal of Cognitive Neuroscience, 11: 80-93, 1999.

BRASS M and VON CRAMON DY. The role of the frontal cortex in task preparation. Cerebral Cortex, 12: 908-914, 2002.

BRAVER TS and BARCH DM. A theory of cognitive control, aging cognition, and neuromodulation. Neuroscience and Biobehavioral Reviews, 26: 809-817, 2002.

Braver TS, Barch DM, Kelley WM, BuckNer RL, COHEN NJ, Miezin FM, SNyder AZ, Ollinger JM, AKbudak E, Conturo TE and Petersen SE. Direct comparison of prefrontal cortex regions engaged by working and long-term memory tasks. NeuroImage, 14: 48-59, 2001

BRAVER TS and BongIOLATTI SR. The role of the frontopolar prefrontal cortex in subgoal processing during working memory. Neurolmage, 16: 317-330, 2002.

BRAVER TS, REYNOLDS JR and DONALDSON DI. Neural mechanisms of transient and sustained cognitive control during task switching. Neuron, 39: 713-726, 2003.

BUCKNER RL. Functional-anatomic correlates of control processes in memory. Journal of Neuroscience, 23: 3999-4004, 2003.

Burgund ED, Lugar HM, Miezin FM and Petersen SE. Sustained and transient activity during an object-naming task: A mixed blocked and event-related fMRI study. NeuroImage, 19: 29-41, 2003

Cabeza R, Dolcos F, Graham R and Nyberg L. Similarities and differences in the neural correlates of episodic memory retrieval and working memory. Neurolmage, 16: 317-330, 2002

Cabeza R, Dolcos F, Prince S, Rice H, Weissman D and NYBERG L. Attention-related activity during episodic memory retrieval: A cross-function fMRI study. Neuropsychologia, 41: 390-399, 2003.

CABEZA R and NyBERG L. Imaging cognition II: Empirical review of 275 PET and fMRI studies. Journal of Cognitive Neuroscience, 12: 1-47, 2000.

Christoff K, REAM JM and Gabrieli JD. Neural basis of spontaneous thought processes. Cortex, 40: 623-630, 2004.

CoHEN JD, Braver TS and O’ReILly R. A computational approach to prefrontal cortex, cognitive control, and schizophrenia: Recent developments and current challenges. Philosophical Transactions of the Royal Society of London B, 351: 15151527, 1996.

Cohen JD, Perlstein WM, Braver TS, Nystrom LE, Noll DC, Jonides $\mathrm{J}$ and SMITH EE. Temporal dynamics of brain activation during a working memory task. Nature, 386: 604608, 1997.

Cohen RM, Semple WE, Gross M, Holcomb HJ, Dowling S and NORDAHL TE. Functional localization of sustained attention. Neuropsychiatry, Neuropsychology and Behavioural Neurology, 1: 3-20, 1988.

Collette $\mathrm{F}$ and VAN DER Linden M. Brain imaging of the central executive component of working memory. Neuroscience and Biobehavioral Reviews, 26: 105-125, 2002.

Coull JT. Neural correlates of attention and arousal: Insights from electrophysiology, functional neuroimaging and psychopharmacology. Progress in Neurobiology, 55: 343-361, 1998.

Coull JT, Frith CD, Frackowiak RS and Grasby PM. A frontoparietal network for rapid visual information processing: A 
PET study of sustained attention and working memory. Neuropsychologia, 34: 1085-1095, 1996.

Courtney SM, Ungerleider LG, KeIL $\mathrm{K}$ and Haxby JV. Transient and sustained activity in a distributed neural system for human working memory. Nature, 386: 608-611, 1997.

CurTis CE and D'Esposito M. Persistent activity in the prefrontal cortex during working memory. Trends in Cognitive Sciences, 7: 415-423, 2003

Curtis CE, Rao VY and D'Esposito M. Maintenance of spatial and motor codes during oculomotor delayed response tasks. Journal of Neuroscience, 24: 3944-3952, 2004.

Desimone R and Duncan J. Neural mechanisms of selective attention. Annual Review of Neuroscience, 18: 193-222, 1995.

Donaldson D, Petersen S, Ollinger J and BuCKNer R. Dissociating state and item components of recognition memory using fMRI. NeuroImage, 13: 129-142, 2001

DONALDSON DI and BUCKNER RL. Effective paradigm design. In Jezzard P, Matthews PM and Smith SM (Eds), Functional MRI: An Introduction to Methods. New York: Oxford University Press, 2001.

Düzel E, Cabeza R, Picton TW, Yonelinas AP, Scheich H, Heinze H-J and Tulving E. Task-related and item-related brain processes of memory retrieval. Proceedings of the National Academy of Sciences of the USA, 96: 1794-1799, 1999.

FRANSSON P. Spontaneous low-frequency BOLD signal fluctuations: An fMRI investigation of the resting-state default mode of brain function hypothesis. Human Brain Mapping, 26: $15-29,2005$

Friston KJ, Fletcher P, Josephs O, Holmes A, RugG MD and TURNER R. Event-related fMRI: Characterizing differential responses. NeuroImage, 7: 30-40, 1998.

Friston KJ, Holmes J, Worsley KJ, Poline JB, Frith CD and FRACKOWIAK RS. Statistical parametric maps in functional imaging: A general linear approach. Human Brain Mapping, 2: $189-210,1995$

FRISTON KJ, PENNY WD and GLASER DE. Conjunction revisited. Neurolmage, 25: 661-667, 2005.

Genovese CR, Lazar NA and Nichols TE. Thresholding of statistical maps in functional neuroimaging using the false discovery rate. NeuroImage, 15: 772-786, 2002.

GHATAN P. Brain activation induced by the perceptual maze test: A PET study of cognitive performance. NeuroImage, 2: 112-124, 1995.

Ghatan P, Hsieh S, Petersson K, Stone-Elander M and Ingvar M. Co-existence of attention based facilitation and inhibition in the human cortex. Neurolmage, 7: 23-29, 1998

Gitelman DR, Nobre AC, Parrish TB, LaBar KS, Kim Y-H, Meyer JR and Mesulam MM. A large-scale distributed network for covert spatial attention: Further anatomical delineation based on stringent behavioural and cognitive controls. Brain, 122: 1093-1106, 1999

Greicius MD, Srivastava G, Reiss AL and Menon V. Defaultmode network activity distinguishes alzheimer's disease from healthy aging: Evidence from functional MRI. Proceedings of the National Academy of Sciences of the USA, 101: 46374642, 2004

Gusnard DA, Akbudak E, Shulman GL and Raichle ME. Medial prefrontal cortex and self-referential mental activity: Relation to a default mode of brain function. Proceedings of the National Academy of Sciences of the USA, 98: 4259-4264, 2001.

GUSNARD DA and RAICHLE ME. Searching for a baseline: Functional imaging and the resting human brain. Nature Neuroscience Reviews, 2: 685-694, 2001.

Henson RN, Shallice T and Dolan RJ. Right prefrontal cortex and episodic memory retrieval: A functional MRI test of the monitoring hypothesis. Brain, 122: 1367-1381, 1999.

HolmES AP and FRISTON KJ. Generalisability, random effects and population inference. NeuroImage, 7: S754, 1998

KIRCHNER WK. Age differences in short-term retention of rapidly changing information. Journal of Experimental Psychology, 55: 352-358, 1958

Koechlin E, Basso G, Pietrini P, Panzer S and Grafman J. The role of the anterior prefrontal cortex in human cognition. Nature, 399: 148-151, 1999.

LaBar KS, Gitelman DR, Parrish TB and Mesulam MM. Neuroanatomic overlap of working memory and spatial attention networks: A functional MRI comparison within subjects. NeuroImage, 10: 695-704, 1999.

Lau HC, Rogers RD, Haggard, P and Passingham RE. Attention to intention. Science, 303: 1208-1210, 2004.

Lepage M, Ghaffar O, Nyberg L and Tulving E. Prefronta cortex and episodic memory retrieval mode. Proceedings of the National Academy of Sciences of the USA, 97: 506-511, 2000 .

LuKs TL, Simpson GV, Feiwell RJ and Miller WL. Evidence for anterior cingulate cortex involvement in monitoring preparatory attentional set. NeuroImage, 17: 792-802, 2002.

MacDonald AW, Cohen JD, Stenger VA and Carter CS Dissociating the role of the dorsolateral prefrontal and anterior cingulate cortex in cognitive control. Science, 288: 1835 $1838,2000$.

MACKWORTH NH. Some factors affecting vigilance. Advanced Sciences, 53: 389-393, 1957.

Macleod AK, Buckner RL, Miezin FM and Petersen SE. Right anterior prefrontal cortex activation during semantic monitoring and working memory. NeuroImage, 7: 41-48, 1998.

MARTIN A. Functional neuroimaging of semantic memory. In Cabeza R and Kingstone A (Eds), Handbook of Functional Neuroimaging of Cognition. Cambridge, MA: MIT Press, 2001.

McKieRnan KA, KaUfman JN, KuCERA-THOMPSON J and Binder JR. A parametric manipulation of factors affecting taskinduced deactivation in functional neuroimaging. Journal of Cognitive Neuroscience, 15: 394-408, 2003.

MILLER EK and COHEN JD. An integrative theory of prefrontal cortex function. Annual Review of Neuroscience, 24: 167-202, 2001

Nichols T, Brett M, Andersson J, Wager T and Poline J-B. Valid conjunction inference with the minimum statistic. NeuroImage, 25: 653-660, 2005.

Nyberg L, Forkstam C, Petersson KM, Cabeza R and Ingvar M. Brain imaging of human memory systems: Betweensystems similarities and within-system differences. Cognitive Brain Research, 13: 281-292, 2002.

Nyberg L, Marklund P, Persson J, Cabeza R, Forkstam C, Petersson K and IngVar M. Common prefrontal activations during working memory, episodic memory, and semantic memory. Neuropsychologia, 41: 371-377, 2003

Nyberg L, Tulving E, Habib R, Nilsson L-G, Kapur S, Houle S and McINTOSH AR. Functional brain maps of retrieval mode and recovery of episodic information. Neuroreport, 7: 249252, 1995 .

Otten LJ, Henson RN and RUGg MD. State-related and itemrelated neural correlates of successful memory encoding. Nature Neuroscience, 5: 1339-1344, 2002.

PARDO JV, Fox PT and RAICHLE ME. Localization of a human system for sustained attention by positron emission tomography. Nature, 349: 61-64, 1991.

Pashler H, Johnston J and RUthruff E. Attention and performance. Annual Review of Psychology, 52: 629-51, 2001

Petersson KM, Sandblom J, Elfgren $C$ and IngVar $M$. Instruction specific brain activations during episodic encoding: A generalized levels of processing effect with visuo-spatial material. NeuroImage, 20: 1795-1810, 2003.

PosNer MI and Petersen SE. The attention system of the human brain. Annual Review of Neuroscience, 13: 25-42, 1990.

Raichle ME, Macleod AK, Snyder AZ, Powers WJ, Gusnard DA and SHULman GL. A default mode of brain function. Proceedings of the National Academy of Sciences of the USA, 98: 676-682, 2001

Ranganath C, COHEN MX and BRozinsky CJ. Working memory maintenance contributes to long-term memory formation: Neural and behavioral evidence. Journal of Cognitive Neuroscience, 17: 994-1010, 2005.

Ranganath C, Cohen MX, Dam C and D'Esposito M. Inferior temporal, prefrontal, and hippocampal contributions to visual working memory maintenance and associative memory retrieval. Journal of Neuroscience, 24: 3917-3925, 2004.

Ranganath C, Johnson M and D'Esposito M. Prefrontal activity associated with working memory and episodic long-term memory. Neuropsychologia, 41: 378-389, 2003.

RugG MD, Fletcher PC, FRITH CD, Frackowiak RS and Dolan NJ. Differential activation of the prefrontal cortex in successful and unsuccessful memory retrieval. Brain, 119 2073-2083, 1996.

SAKAI K and PASSINGHAM RE. Prefrontal interactions reflect future task operations. Nature Neuroscience, 6: 75-81, 2003.

Shulman GL, Fiez JA, Corbetta M, Buckner RL, Miezin FM, Raichle ME and Petersen SE. Common blood flow changes across visual tasks: II. Decreases in cerebral cortex. Journal of Cognitive Neuroscience, 9: 648-663, 1997.

SMITH EE and JoNIDES J. Working memory: A view from neuroimaging. Cognitive Psychology, 33: 5-42, 1997. 
TAlairach J and Tournoux P. Co-Planar Stereotaxic Atlas of the Human Brain. Stuttgart: Thieme, 1988.

THOMPSON-SCHILL SL. Neuroimaging studies of semantic memory: Inferring "how" from "where". Neuropsychologia, 41: 280292, 2003.

Thompson-SChill SL, D'Esposito M and Kan IP. Effects of repetition and competition on activity in left prefrontal cortex during word generation. Neuron, 23: 513-522, 1999.

Tulving E, Kapur S, Markowitsch HJ, Craik FI, Habib R and HOULE S. Neuroanatomical correlates of retrieval in episodic memory: Auditory sentence recognition. Proceedings of the National Academy of Sciences of the USA, 91: 2012-2015, 1994.

Velanova K, Jacoby Ll, WheELER ME, McAvoy MP, Petersen SE and BUCKNER RL. Functional-anatomic correlates of sustained and transient processing components engaged during controlled retrieval. Journal of Neuroscience, 23 : 8460-8470, 2003.

Visscher KM, Miezin FM, Kelly JE, BuCKNer RL, Donaldson
DI, McAvoy MP, Bhalodia VM and Petersen SE. Mixed blocked/event-related designs separate transient and sustained activity in fMRI. NeuroImage, 19: 1694-1708, 2003.

WAGNER AD. Working memory contributions to human learning and remembering. Neuron, 22: 19-22, 1999.

WAGNER AD. Cognitive control and episodic memory: Contributions from prefrontal cortex. In Squire LR and Schacter DL (Eds), Neuropsychology of Memory. New York: The Guilford Press, 2001.

Wagner AD, Paré-Blagoev EJ, Clark J and Poldrack RA. Recovering meaning: Left prefrontal cortex guides controlled semantic retrieval. Neuron, 31: 329-338, 2001.

WHEELER MA and BUCKNER RL. Functional dissociation among components of remembering: Control, perceived oldness, and content. Journal of Neuroscience, 23: 3869-3880, 2003

Petter Marklund, Department of Psychology, Umeå University, S-901 87 Umeå, Sweden. e-mail: Petter.Marklund@ psy.umu.se

(Received 2 March 2005; reviewed 1 August 2005; revised 26 October 2005; accepted 13 February 2006) 